\title{
Uniqueness of the Cauchy problem for convolution equations
}

\author{
By Sönke Hansen at Paderborn
}

\section{Introduction}

In the study of the Cauchy problem for a convolution equation with Cauchy data on an initial hyperplane or strip one encounters the following uniqueness problem. Let $0 \neq \mu \in \mathscr{E}^{\prime}\left(\mathbb{R}^{n}\right)$ and let $N \in \mathbb{R}^{n}, N \neq 0$. Under what conditions on $\mu$ and $N$ does every distribution $u \in \mathscr{D}^{\prime}\left(\mathbb{R}^{n}\right)$, which has its support contained in the half space

$$
\{x ;\langle x, N\rangle \geqq 0\}
$$

and is a solution to the convolution equation

$$
\mu * u=0 \quad \text { in } \mathbb{R}^{n},
$$

already vanish in the whole space? Since this problem only involves the homogeneous convolution equation it is natural to try to express the looked-for conditions in terms of $N$ and the zeros of the Fourier transform $\hat{\mu}$ of $\mu$.

In case (1. 1) is a partial differential equation with constant coefficients, i.e. $\mu=P(D) \delta$ for some non-zero polynomial $P$, this problem can be solved completely with the help of the notion of a characteristic vector, which is by definition a zero of the principal part $P_{m}$ of $P$. In fact, we have by Holmgren's uniqueness theorem and by Theorem 5. 2. 2. in Hörmander [7]: There exists a non-vanishing distribution $u \in \mathscr{D}^{\prime}\left(\mathbb{R}^{n}\right)$ satisfying the equation $P(D) u=0$ and having its support contained in $\{x ;\langle x, N\rangle \geqq 0\}$ if and only if $N$ is a characteristic vector for $P$.

In Definition 2. 1. we give a generalization of the notion of a characteristic vector to arbitrary distributions $\mu \in \mathscr{E}^{\prime}\left(\mathbb{R}^{n}\right)$. Using this definition we then extend Holmgren's uniqueness theorem to convolution equations in Theorem 2. 2. This is our main result. The idea of the proof of Theorem 2. 2. was suggested to the author by Ehrenpreis' method of proving Holmgren's uniqueness theorem for partial differential equations with constant coefficients. Ehrenpreis [4] proves this theorem via Fourier analysis and not with the help of the Cauchy-Kovalerska theorem as is often done. Originally (i.e. in Hansen [6]) we were only able to prove a weaker version of Theorem 2.2, for invertible (in the sense of Ehrenpreis [2]) distributions $\mu$ since we were not aware of the approximation technique developed in Hörmander [9] then. 
A refined uniqueness theorem (Theorem 3. 2.) is proved in section 3 for differentialdifference equations, i.e. equations (1.1) with $\mu$ having finite support. Here we give for two convex domains $\Omega^{1}$ and $\Omega^{2}$ with $\Omega^{1} \subset \Omega^{2}$ sufficient conditions, mainly in terms of $\Omega^{1}, \Omega^{2}$ and the set of characteristic vectors for $\mu$, such that any solution $u$ of the equation (1. 1) defined by $\mu$ and vanishing in $\Omega^{1}$ already vanishes in the larger domain $\Omega^{2}$.

In section 4 we shall first show (Proposition 4. 1.) that our definition of a characteristic vector $N$ for a convolutor $\mu$ (Definition 2.1.) is indeed a generalization of the classical definition $P_{m}(N)=0$ for differential operators $\mu=P(D) \delta$, for the condition $P_{m}(N)=0$ is in fact a condition on the set of zeros of $P$. Furthermore we obtain a nonuniqueness theorem for characteristic half spaces (Theorem 4. 2.). Together with Theorem 2.2. these results show that for a convolution equation (1. 1) defined by $\mu=P(D) \delta+v$ with $P$ a non-zero polynomial and $v \in \mathscr{E}^{\prime}\left(\mathbb{R}^{n}\right)$, supp $v \Subset\{x ;\langle x, N\rangle>0\}$, the answer to the uniqueness problem stated at the beginning of the introduction and the property that $N$ be a characteristic vector for $\mu$ are independent of $v$. That this result should become true was our guideline to the definition of a characteristic vector. However, it is not clear to us whether it is true for every $0 \neq \mu \in \mathscr{E}^{\prime}\left(R^{n}\right)$ that all solutions $u$ of (1. 1) with supp $u \subset\{x ;\langle x, N\rangle \geqq 0\}$ vanish in $R^{n}$ if and only if $N$ is a non-characteristic vector for $\mu$ in the sense of Definition 2.1 .

The notation we employ is that of Hörmander [7] and [8]. For $0 \neq \mu \in \mathscr{E}^{\prime}\left(\mathbb{R}^{n}\right)$ we denote by $H_{\mu}$ the supporting function of supp $\mu$. Having introduced coordinates we shall use the splitting of variables $x=\left(x^{\prime}, x_{n}\right)$ and $z=\left(z^{\prime}, z_{n}\right)$ with $x^{\prime} \in \mathbb{R}^{n-1}, x_{n} \in \mathbb{R}$, and $z^{\prime} \in \mathbb{C}^{n-1} . z_{n} \in \mathbb{C}$. for $x \in \mathbb{R}^{n}$ and $z \in \mathbb{C}^{n}$, respectively. $\langle\cdot, \cdot\rangle$ denotes both the duality bracket or the euclidean product (when coordinates are used) on $R^{n}$ (or $\mathbb{C}^{n}$ ) depending on whether the arguments are dual vectors or not.

I would like to thank Prof. Dr. J. Wloka who suggested this work to me and Dr. O. von Grudzinski who read an earlier version of this paper and gave me suggestions
for improvements.

\section{Uniqueness of the non-characteristic Cauchy problem}

We start with the definition of a (non-)characteristic vector.

Definition 2. 1. Let $n>1$ and let $N \in \dot{R}^{n}, \mu \in \mathscr{E}^{\prime}\left(\mathbb{R}^{n}\right) . N$ is called a non-characteristic rector for $\mu$ if and only if there is a constant $b>0$ such that the sets

$$
E(b, \delta) \cap\left\{z \in \mathbb{C}^{n} ; \hat{\mu}(-z)=0\right\}
$$

are bounded for every $\delta>0$. Here $E(b, \delta)$ is defined for $b, \delta>0$ and some choice of

$$
E(b, \delta)=\left\{z \in \mathbb{C}^{n} ;\left|z_{n}\right| \geqq b|\operatorname{Rez}|+\delta|z|, \operatorname{Imz}_{n} \geqq b|\operatorname{Imz}|+\delta|z|\right\} .
$$

Non-zero vectors in the complement of the set of all non-characteristic vectors for $\mu$ are
said to be characteristic vectors for $\mu$. 
Remark. An elementary calculation shows that the sets $E(b, \delta) \cap\left\{z \in \mathbb{C}^{n} ; \hat{\mu}(-z)=0\right\}$ are bounded for some $b>0$ and every $\delta>0$ if and only if the sets

$$
\left\{z \in \mathbb{C}^{n} ; \hat{\mu}(-z)=0,\left|z_{n}\right| \geqq c\left|z^{\prime}\right|, \operatorname{Imz}_{n} \geqq c\left|\operatorname{Imz}^{\prime}\right|+\delta|=|\right\}
$$

are bounded for some $c>0$ and every $\delta>0$. Starting from this observation it is easy to check that the definition of a non-characteristic vector is independent of the particular choice of coordinates in $\mathbb{C}^{n}$ with $N=(0, \ldots, 0,1)$ and which are real on $\mathbb{R}^{n}$.

It will be shown in Proposition 4. 1 below that our definition of a (non-)characteristic vector for $\mu \in \mathscr{E}^{\prime}\left(\mathbb{R}^{n}\right)$ agrees with the classical definition in case $\mu=P(D) \delta$ for some nonzero polynomial $P$. Hence the following result extends Holmgren's uniqueness theorem from linear partial differential equations with constant coefficients to convolution equations.

Theorem 2. 2. Let $n>1$. Let $N \in \dot{R}^{n}$ be a non-characteristic vector for $\mu \in \mathscr{E}^{\prime}\left(\mathbb{R}^{n}\right)$. Then every $u \in \mathscr{L}^{\prime}\left(\mathbb{R}^{n}\right)$ canishing in the half space $\{x ;\langle x, N\rangle<0\}$ and satisfiving $\mu * u=0$ in $\mathbb{R}^{n}$ vanishes in $\mathbb{R}^{n}$.

Remark. For any non-zero $\mu \in \mathscr{E}^{\prime}\left(\mathbb{R}^{1}\right)$ the uniqueness statement in the preceding theorem is valid in view of Titchmarsh's theorem on supports.

Remark. If $\mu$ is (semi-)hyperbolic with respect to $N$ in the sense of Ehrenpreis [3] then $N$ is a non-characteristic vector for $\mu$. Hence Theorem 2. 2. in particular covers the uniqueness statement for hyperbolic Cauchy problems.

The proof of Theorem 2. 2. will be given at the end of this section. It may be useful for the reader to look at it first to obtain a motivation for the following preparations.

The following lemma is essentially the Lemma 9. 22. in Ehrenpreis [4].

Lemma 2. 3. Let $n>1$. Let $a, b, t$ real and $a, b>0$. Define the set

$$
\omega=\left\{x \in \mathbb{R}^{n} ;\left|x^{\prime}\right|<a, 0<x_{n}-t<(2 a b)^{-1}\left(a^{2}-\left|x^{\prime}\right|^{2}\right)\right\} .
$$

Let $u$ be a $C^{x}$-finction defined in a neighbourhood of $\overline{0}$ such that $v(u)=0$ for erery $v \in \mathscr{E}^{\prime \prime}(\bar{D})$ which satisfies for some $C>0$ and every $\varepsilon>0$

(2. 2) $|\hat{v}(z)| \leqq C \exp \left(a\left|\operatorname{Im} z^{\prime}\right|+t \operatorname{Im} z_{n}+\varepsilon|z|\right), z \in \mathbb{C}^{n} \backslash E(b, b \varepsilon a)$.

Then uramishes in $\omega$.

Proof. Let $\beta>b$ and let $P$ be a non-zero polynomial in $n$ variables. Define $v \in \delta^{\prime}(\overline{1})$ by

$$
v(v)=\int_{|\xi| \leqq a}(P t)\left(\xi, t+(2 \alpha \beta)^{-1}\left(a^{2}-|\xi|^{2}\right)\right) d \xi, t \in C^{3}\left(\mathbb{R}^{n}\right) .
$$

We claim that $v$ satisfies (2. 2) for some $C>0$ and every $\varepsilon>0$. Once this is shown we have $v(u)=0$ by the hypothesis of Lemma 2 . 3. Letting $P$ vary we may then conclude by the Stone-Weierstra $B$ theorem that the restriction of $u$ to the parabolic surface

$$
\left\{\left(\xi, t+(2 a \beta)^{-1}\left(a^{2}-|\xi|^{2}\right)\right) ; \xi \in \mathbb{R}^{n-1},|\xi| \leqq a\right\}
$$

vanishes. Letting $\beta>b$ vary, we would then obtain $u=0$ in $\omega$, as desired. 
The Fourier transform $\hat{v}$ of $v$ defined in (2.3) reads

(2. 4)

$$
\hat{v}(z)=e^{-i t z_{n}} \int_{\Gamma} P\left(\zeta, t+(2 a \beta)^{-1}\left(a^{2}-\langle\zeta, \zeta\rangle\right)\right) \exp \left(-i\left\langle z^{\prime}, \zeta\right\rangle-i z_{n}(2 a \beta)^{-1}\left(a^{2}-\langle\zeta, \zeta\rangle\right)\right) d \zeta
$$

for $z \in \mathbb{C}^{n}$. Here $\Gamma$ is the $(n-1)$-chain defined by $\Gamma(\xi)=\xi,|\xi| \leqq a, \xi \in \mathbb{R}^{n-1}$. Let $\varepsilon>0$. By (2.1) we have either $\operatorname{Im} z_{n}<b\left|\operatorname{Im} z^{\prime}\right|+b \varepsilon|z| / a$ or $\left|z_{n}\right|<b\left|\operatorname{Re} z^{\prime}\right|+b \varepsilon|z| / a$ if $z \in \mathbb{C}^{n} \backslash E(b, b \varepsilon / a)$. Let $z \in \mathbb{C}^{n}$ satisfy $\operatorname{Im} z_{n}<b\left|\operatorname{Im} z^{\prime}\right|+b \varepsilon|z| / a$. Using the Cauchy-Schwarz inequality we obtain for $\xi \in \mathbb{R}^{n-1},|\xi| \leqq a$,

$$
\begin{aligned}
\operatorname{Re}\left(-i\left\langle z^{\prime}, \xi\right\rangle-i z_{n}(2 a \beta)^{-1}\left(a^{2}-|\xi|^{2}\right)\right) \\
\quad<\left|\operatorname{Im} z^{\prime}\right|\left(|\xi|+b(2 a \beta)^{-1}\left(a^{2}-|\xi|^{2}\right)\right)+\left(2 a^{2}\right)^{-1} \varepsilon|z|\left(a^{2}-|\xi|^{2}\right)<a\left|\operatorname{Im} z^{\prime}\right|+\varepsilon|z| .
\end{aligned}
$$

Estimating the integral (2.4) we hence obtain the inequality (2.2) with a constant $C$ independent of $\varepsilon$ and $z$. Now let $z \in \mathbb{C}^{n}$ satisfy $\left|z_{n}\right|<b\left|\operatorname{Re} z^{\prime}\right|+b \varepsilon|z| / a, \operatorname{Re} z^{\prime} \neq 0$. If $\xi, \eta \in \mathbb{R}^{n-1}$ are such that $|\xi| \leqq a,\left\langle\operatorname{Re} z^{\prime}, \eta\right\rangle=-\left|\operatorname{Re} z^{\prime}\right||\eta|,|\eta|=\left(a^{2}-|\xi|^{2}\right)^{\frac{1}{2}}$, we have, using the Cauchy-
Schwarz inequality,

$$
\begin{aligned}
\operatorname{Re}(- & \left.i\left\langle z^{\prime}, \xi+i \eta\right\rangle-i z_{n}(2 a \beta)^{-1}\left(a^{2}-\langle\xi+i \eta, \xi+i \eta\rangle\right)\right) \\
& \leqq a\left|\operatorname{Im} z^{\prime}\right|-\left|\operatorname{Re} z^{\prime}\right||\eta|+\left.\left|z_{n}\right||2| \eta\right|^{2}-2 i\langle\xi, \eta\rangle \mid(2 a \beta)^{-1} \\
& <a\left|\operatorname{Im} z^{\prime}\right|-\left|\operatorname{Re} z^{\prime}\right|\left(|\eta|-|\eta||2 \eta-2 i \xi| b(2 a \beta)^{-1}\right)+|z||\eta||\eta-i \xi| \varepsilon a^{-2} \\
& \leqq a\left|\operatorname{Im} z^{\prime}\right|+\varepsilon|z| .
\end{aligned}
$$

Since the integrand in (2.4) is a holomorphic $(n-1)$-form we may by Stokes' theorem replace the chain of integration in (2.4) by the chain $\Gamma$ defined by

$$
\Gamma(\xi)=\xi-i\left(a^{2}-|\xi|^{2}\right)^{\frac{1}{2}} \eta_{0}, \xi \in \mathbb{R}^{n-1},|\xi|<a,
$$

and $\eta_{0} \in \mathbb{R}^{n-1} \cdot\left|\eta_{0}\right|=1,\left\langle\eta_{0} ., \operatorname{Re} z^{\prime}\right\rangle=\left|\operatorname{Re} z^{\prime}\right|$ fixed. Estimating this integral we obtain, since the foregoing estimates are valid on $\Gamma$. the inequality (2.2) with a constant $C$ independent
of $\varepsilon$ and $z$. This proves Lemma 2.3

Next we need a lower estimate of the modulus of $\hat{\mu}, \mu \in \mathscr{E}^{\prime \prime}\left(\mathbb{R}^{n}\right)$, off its set of zeros. Lemma 2. 4. Let $\mu \in \mathscr{E}^{\prime}\left(\mathbb{R}^{n}\right)$ and let $a>0$. Then there is a real number $A$ such that
we hare, for arbitrary $\varepsilon>0$

$d(z)$ denotes the distance from $z+10$. where $C$ is a positice constant independent of $z$. Here

Proof. (Compare Hörmander [9], Lemma 2. 3.) By the Paley-Wiener theorem the
plurisubharmonic function $v, v(z)=\log |\hat{\mu}(z)|$, satisfies for

$$
\begin{aligned}
& v(z) \leqq C_{0}+C_{1}|\operatorname{Im} z|+\gamma|z|, z \in \mathbb{C}^{n} .
\end{aligned}
$$


With $\mathbf{M}$ and $\mathbf{M}^{r}$ denoting mean value with respect to Lebesgue measure in $\mathbb{C}^{n}$ and in $\mathbb{R}^{n}$. respectively, we have since $v$ is subharmonic

$$
v(x) \leqq \underset{|z-x|<R}{\mathbf{M}} v(z) \leqq \underset{|z-x|<R}{\mathbf{M}}\left(v(z)-C_{0}-C_{1}|\operatorname{Im} z|-\gamma|z|\right)+C_{0}+\left(C_{1}+\gamma\right) R+\gamma|\cdot x| .
$$

Since

$$
\underset{|z-x|<R}{\mathbf{M}}|v(z)| \leqq C_{0}+\left(C_{1}+\gamma\right) R+\gamma|x|+\underset{|z-x|<R}{\mathbf{M}}\left(C_{0}+C_{1}|\operatorname{Im} z|+\gamma|z|-v(z)\right)
$$

it follows that

$$
\underset{|z-x|<R}{\mathbf{M}}|v(z)| \leq 2\left(C_{0}+\left(C_{1}+\gamma\right) R+\gamma|x|\right)+|v(x)| .
$$

Now replace $x$ by $x^{\prime}$ and average with respect to Lebesgue measure in $\mathbb{R}^{n}$ over all $x^{\prime} \in \mathbb{R}^{n}$ with $\left|x-x^{\prime}\right|<R / 2$. It follows that

$$
\text { (2. 6) } 2^{-2 n} \underset{|z-x|<R / 2}{\mathbf{M}}|v(z)| \leqq 2\left(C_{0}+\left(C_{1}+2 \gamma\right) R+\gamma|x|\right)+\underset{\left|x-x^{\prime}\right|<R / 2}{\mathbf{M}^{r}}\left|v\left(x^{\prime}\right)\right| .
$$

Assume without loss of generality $a \leqq 1$. Let $z \in \mathbb{C}^{n}$ be such that $d(z) \geqq a R / 4$ and $|z-x|<R / 4$. Hence we may apply the mean value theorem to $-v$ to give

and therefore using (2.6)

$$
-v(z) \leqq \underset{\left|z-z^{\prime}\right|<a R / 4}{\mathbf{M}}\left|v\left(z^{\prime}\right)\right| \leqq(2 / a)^{2 n} \underset{\left|z^{\prime}-x\right|<R / 2}{\mathbf{M}}\left|v\left(z^{\prime}\right)\right|
$$

$$
-(4 / a)^{-2 n} v(z) \leqq 2\left(C_{0}+\left(C_{1}+2 \gamma\right) R+y|x|\right)+\underset{\left|x-x^{\prime}\right|<R / 2}{\mathbf{M}^{r}}\left|v\left(x^{\prime}\right)\right| .
$$

Let $\delta>0$ and choose $x=\operatorname{Re} z, R=4|\operatorname{Im} z|+4 \delta|\operatorname{Re} z| / a$. It follows from Lemma 2.2. in Hörmander [9] that

$$
t^{-1} \underset{|y|<t}{\mathbf{M}^{r}}|v(y)| \rightarrow 0 \quad \text { as } \quad t \rightarrow+\infty
$$

(note, that with the help of a mollifier one can easily get rid of the condition (2.1) appearing there). Setting $t=|x|+R$ we therefore deduce using $t \leqq\left(1+a(4 \delta)^{-1}\right) R$ that the average last written in (2.7) is $o(|z|)$ when $z \rightarrow \chi$. So we conclude that (2.5) is valid for $\varepsilon=\max \left(\delta, 3(4 / a)^{2 n}\left(\left(C_{1}+2 \gamma\right) 4 \delta / a+\gamma\right)\right)$ and $A=8(4 / a)^{2 n}\left(C_{1}+2\right)$. This proves the Lemma.

With the help of the following theorem we can approximate entire functions of exponential type by entire functions which are in view of the Paley-Wiener theorem Fourier transforms of distributions with compact support.

Theorem 2. 5. Let $F_{1}, F_{2}, G_{1}$ and $G_{2}$ be entire functions such that $G_{1}=F_{1}+G_{2} F_{2}$. Assume that there are supporting functions $H_{1}$ and $H_{2}$ such that

$$
\left|F_{i}(z)\right| \leqq C \exp H_{i}(\operatorname{Im} z), 2|\operatorname{Im} z|>|\operatorname{Re} z|, i=1,2,
$$

and that $F_{1}$ and $F_{2}$ are of exponential type in the whole space,

$$
\left|F_{i}(z)\right| \leqq C e^{A|z|}, z \in \mathbb{C}^{n}, \quad i=1,2 .
$$


For $i=1.2$ and $0<t<1$ we then can find entire functions $F_{i}^{t}$ such that for all $z \in \mathbb{C}^{n}$

$$
\left|F_{i}^{t}(z)\right| \leqq C(t)(1+|z|)^{n+6} \exp H_{i}(\operatorname{Im} z)
$$

and

$$
\begin{aligned}
\mid G_{1}(z) & -F_{1}^{t}(z)-G_{2}(z) F_{2}^{t}(z) \mid \\
& \leqq C t\left(\left|G_{1}(z)\right||z|^{2}+(1+|z|)^{n+6} \exp H_{1}(\operatorname{Im} z)+\left|G_{2}(z)\right|(1+|z|)^{n+6} \exp H_{2}(\operatorname{Im} z)\right) .
\end{aligned}
$$

It is clear from the remark following the proof of Theorem 3. 1. in Hörmander [9] that the proof of that Theorem immediately extends to a proof of Theorem 2.5.

We now turn to the

Proof of the Theorem 2. 2. In view of the standard technique of regularisation with a Dirac sequence it suffices to prove the statement of the theorem for $u \in C^{x}\left(\mathbb{R}^{n}\right)$. Choose coordinates such that $N=(0, \ldots, 0,1)$. By the definition of a non-characteristic vector there is a positive constant $b$ such that the set

$$
E(b / 2, \delta) \cap\left\{z \in \mathbb{C}^{n} ; \hat{\mu}(-z)=0\right\}
$$

is bounded for every $\delta>0\left(E\right.$ is defined in (2.1)). Let $a>0$ and $v \in \mathscr{E}^{\prime}\left(\mathbb{R}^{n}\right)$ such that

$$
|\hat{v}(z)| \leqq C \exp \left(a\left|\operatorname{Im} z^{\prime}\right|-(4 b)^{-1} a \operatorname{Im} z_{n}+|z|\right)
$$

if $z \in C_{n} \backslash E(b . b(a)$ for some constant $C$ independent of $z$. We claim that $v(u)=0$ if $a$ is sufficiently large. From Lemma 2. 3. we then deduce

$$
u(x)=0 \text { if }\left|x^{\prime}\right|<a \text { and }-a(4 b)^{-1}<x_{n}<(2 a b)^{-1}\left(a^{2} / 2-\left|x^{\prime}\right|^{2}\right) .
$$

After letting $a \rightarrow+x_{-}$we then obtain the statement of the theorem.

To prove $v(u)=0$ we shall approximate $v \in \mathscr{E}^{\prime}\left(\mathbb{R}^{n}\right)$ by distributions of the form $\sigma+\ddot{\mu} * \tau$. Where $\sigma . \tau \in \delta^{\prime}\left(\mathbb{R}^{n}\right)$. supp $\sigma \Subset\{x ;\langle x, N\rangle<0\}$, because for these distributions we hatte $(\sigma+\ddot{\mu} * \tau)(u)=0$. Working on the Fourier transform side we therefore set for $z \in \mathbb{C}^{n}$

(2. 10$)$

(2. 11) $T(z)=(1-\varphi(z)) \hat{n}(z) \hat{\mu}(-z)+\iota(z)$.

with functions $\varphi \cdot l \in L_{1, c}^{2}\left(C^{\prime \prime}\right)$ such that $\varphi=1$ in a neighbourhood of the set of zeros of $\tilde{\mu}$.

$$
\hat{r}(z)=S(z)+\hat{\mu}(-z) T(z) . \quad z \in \mathbb{C}^{n} .
$$

To make $S$ and $T$ analytic we have to choose $r$ as a solution of

$$
\bar{i}(こ)=g(z) . \quad z \in \mathbb{C}^{n} \text {. }
$$

with the (0. 1)-form $g$ defined by

$$
g(z)=(\hat{v}(z) \hat{\mu}(-z)) \overline{\hat{c}} \varphi(z), z \in \mathbb{C}^{n} .
$$

But first we want to choose $\varphi$ so that the modulus of $g$ satisfies good estimates. Let $\delta>0$ and let $z \in E(b, \delta)$. Note that $\left(1+b^{-1}\right)^{-1}|\operatorname{Im} z| \leqq \operatorname{Im} z_{n}$. Let $\zeta \in \mathbb{C}^{n}$ satisfy

$$
|\zeta| \leqq(1+b ; 2)^{-1}\left(\left(2+2 b^{-1}\right)^{-1}|\operatorname{Im} z|+\delta|z| / 4\right) \text {. }
$$


Then we have

$$
\begin{aligned}
\operatorname{Im}\left(z_{n}+\check{\zeta}_{n}\right) & \geqq b\left|\operatorname{Im} z^{\prime}\right| 12+\delta|=| 12+\operatorname{Im}=z_{n}|2-| \zeta \mid \\
& \geqq b\left|\operatorname{Im}\left(z^{\prime}+\zeta^{\prime}\right)\right| / 2+\delta|=| / 2+\left(2+2 b^{-1}\right)^{-1}|\operatorname{Im} z|-(1+b / 2)|\zeta| \\
& \geqq b\left|\operatorname{Im}\left(z^{\prime}+\zeta^{\prime}\right)\right| / 2+\delta|=| 4
\end{aligned}
$$

and after a similar calculation,

$$
\left|z_{n}+\zeta_{n}\right| \geqq b\left|\operatorname{Re}\left(z^{\prime}+\zeta^{\prime}\right)\right| 2+\delta|=| 4
$$

In view of $(2.8)$ and because $|\xi|<(2+\delta)|=| / 4$ we therefore obtain that the distance from $-z$ to the set of zeros of $\hat{\mu}$ is greater than $(1+b)^{-1}\left(\left(2+2 b^{-1}\right)^{-1}|\operatorname{Im}=|+\delta|=| 4\right)$ for sufficiently large $=$ if $\delta<2$. From Lemma 2. 4. it then follows that there is a constant $A \geqq 0$ depending only on $b$ such that for $\delta<2$

$$
|\hat{\mu}(-z)| \geqq \exp (-A|\operatorname{Im}=|-|z|) \quad \text { if } \quad z \in E(b, \delta),|=| \geqq C(\dot{\delta}) .
$$

Choosing $\delta<\min (2, b / a)$ and taking $\varphi \in C^{x}\left(C^{n}\right)$ for example as the convolution product of the characteristic function of the set

$$
\left\{z \in \mathbb{C}^{n}:|z| \leqq 1+C(\delta)\right\} \cup\left(\mathbb{C}^{n} \backslash E(b,(\delta+b, a) 2)\right)
$$

and a $C^{x}$-function having its support contained in a sufficiently small neighbourhood of zero and having Lebesgue integral equal to one we obtain a function $\varphi$ such that (2.9) is valid in $\operatorname{supp} \varphi$. (2.15) is valid in $\operatorname{supp}(1-\varphi)$ and $|\overline{\hat{c}} \varphi|$ is bounded on $\mathbb{C}^{n}$. Using this function $\varphi$ we define $g$ by $(2.14)$ and obtain

$$
|g(z)| \leqq C \exp (H(\operatorname{Im} z)+A|\operatorname{Im} z|+2|z|) .=\in \mathbb{C}^{n} .
$$

Here $H$ is the supporting function defined by

$$
H(\eta)=a\left|\eta^{\prime}\right|-a(4 h)^{-1} \eta_{n}, \quad \eta \in \mathbb{R}^{n}
$$

Since the right-hand side in (2.16) is a logarithmically plurisubharmonic function it follows from Theorem 4. 4. 2. in Hörmander [8] that the equation (2. 13) has a solution $l \in L_{\text {low }}^{2}\left(\mathbb{C}^{n}\right)$ such that

$$
\text { (2. 18) } \int_{n}|f(z)|^{2} \exp (-2 H(\operatorname{Im}=)-2 A|\operatorname{Im}=|-5|=|) d \lambda(z)<+3 .
$$

where $d$ denotes Lebesgue measure in $\mathbb{C}^{n}$. Combining (2.9). (2. 10). (2.11). (2.15) and (2. 18) we obtatin

$$
S \exp (-H(\operatorname{Im} \cdot)-(A+B)|\operatorname{Im} \cdot|-3|\cdot|) \in L^{2}\left(C^{n}\right)
$$

and

$$
T \exp (-(A+B)|\operatorname{Im} \cdot|-3|\cdot|) \in L^{2}\left(\mathbb{C}^{n}\right)
$$


Here we have chosen $B>0$ such that $H(\eta) \leqq B|\eta|, \eta \in \mathbb{R}^{n}$, and

$$
\max (|\hat{\mu}(z)|,|\hat{v}(z)|) \leqq C \exp (B|\operatorname{Im} z|+|z|), z \in \mathbb{C}^{n},
$$

which is possible in view of the Paley-Wiener theorem. Since $S$ and $T$ are entire analytic functions it follows by Cauchy's formula that with the supporting functions

$$
H_{1}=H+(A+B+9)|\cdot|, \quad H_{2}=(A+B+9)|\cdot|
$$

we have for all $z \in \mathbb{C}^{n}$

$$
\begin{aligned}
& |S(z)| \leqq C \exp \left(H_{1}(\operatorname{Im} z)+3(|\operatorname{Re} z|-2|\operatorname{Im} z|)\right) \\
& |T(z)| \leqq C \exp \left(H_{2}(\operatorname{Im} z)+3(|\operatorname{Re} z|-2|\operatorname{Im} z|)\right) .
\end{aligned}
$$

This means in particular that $F_{1}:=S, F_{2}:=T$ and $G_{2}:=\hat{\mu}$ satisfy the assumptions of Theorem 2. 5. Hence there exist entire functions $S_{t}$ and $T_{t}, 0<t<1$, such that for all $z \in \mathbb{C}^{n}$

$$
\begin{aligned}
& \left|S_{t}(z)\right| \leqq C(t)(1+|z|)^{n+6} \exp H_{1}(\operatorname{Im} z), \\
& \left|T_{t}(z)\right| \leqq C(t)(1+|z|)^{n+6} \exp H_{2}(\operatorname{Im} z),
\end{aligned}
$$

and we have for some constants $C$ and $M$

$$
\left|\hat{v}(\xi)-S_{t}(\zeta)-\hat{\mu}(-\xi) T_{t}(\xi)\right| \leqq C t(1+|\xi|)^{M}, \xi \in \mathbb{R}^{n} .
$$

Here we have used (2.12) and the fact that $\hat{v}$ and $\hat{\mu}$ are of polynomial growth in $\mathbb{R}^{n}$. By the Paley-Wiener theorem and (2.20), (2.21) there exist distributions $\sigma_{t}, \tau_{t} \in \mathscr{E}^{\prime}\left(\mathbb{R}^{n}\right)$ such that $\hat{\sigma}_{t}=S_{t}$ and $\hat{\tau}_{t}=S_{t}$. If $a$ satisfies $a>4 b(A+B+9)$ then $H_{1}(N)<0$ in view of (2. 17). (2. 19). and hence supp $\sigma_{t} \Subset\{x ;\langle x, N\rangle<0\}$ by (2. 20) and the Paley-Wiener theorem. From (2. 22) it follows that $\sigma_{t}+\check{\mu} * \tau_{t} \rightarrow v$ as $t \rightarrow 0$ in $\mathscr{S}^{\prime}$. In view of (2.20), (2.21) and the Paley-Wiener theorem the support of $\sigma_{t}+\check{\mu} * \tau_{t}$ belongs to a fixed compact subset of $\mathbb{R}^{n}$ so that we have in fact convergence in $\mathscr{E}^{\prime}\left(\mathbb{R}^{n}\right)$. If $u \in C^{\infty}\left(\mathbb{R}^{n}\right)$ satisfies $\mu * u=0$ and has its support contained in $\{x ;\langle x, N\rangle \geqq 0\}$ we therefore obtain

$$
v(u)=\lim _{t \rightarrow 0}\left(\sigma_{t}+\check{\mu} * \tau_{t}\right)(u)=\lim _{t \rightarrow 0}\left(\sigma_{t}(u)+\tau_{t}(\mu * u)\right)=0 .
$$

It has already been pointed out that this completes the proof of Theorem 2. 2.

Example. Let $\mu$ be the distribution defined by the characteristic function of the unit ball given by some positive definite metric on $\mathbb{R}^{n}$. We shall show that every $N \in \mathbb{R}^{n}$ is non-characteristic for $\mu$. In view of the remark following Definition 2.1. we may introduce coordinates in $\mathbb{R}^{n}$ such that the metric is euclidean and $N=(0, \ldots, 0,1)$. As is
well known (see e.g. Bochner [1], p. 235) the Fourier transform of $\mu$ is given by

$$
\hat{\mu}(z)=C_{n}\langle z, z\rangle^{-\frac{n}{4}} \mathrm{~J}_{\frac{n}{2}}\left(\langle z, z\rangle^{\frac{1}{2}}\right), z \in \mathbb{C}^{n} .
$$


Here $\mathrm{J}_{\frac{n}{2}}$ is the Bessel function of order $\frac{n}{2}$. Let $z \in \mathbb{C}^{n}$ with $\hat{\mu}(z)=0$. Since $\mathrm{J}_{\frac{n}{2}}$ has only real zeros (see e.g. Watson [10], p. 482) we have

and hence

$$
0 \leqq\langle z, z\rangle=|\operatorname{Re} z|^{2}-|\operatorname{Im} z|^{2}+2 i\langle\operatorname{Re} z, \operatorname{Im} z\rangle
$$

$$
\begin{gathered}
|\operatorname{Im} z| \leqq|\operatorname{Re} z|, \\
\left|\operatorname{Re} z_{n}\right|\left|\operatorname{Im} z_{n}\right| \leqq\left|\operatorname{Re} z^{\prime}\right|\left|\operatorname{Im} z^{\prime}\right| .
\end{gathered}
$$

Using (2. 23) we have if $\left|\operatorname{Re} z_{n}\right| \leqq\left|\operatorname{Re} z^{\prime}\right|$

$$
\left|z_{n}\right| \leqq\left|\operatorname{Re} z_{n}\right|+\left|\operatorname{Im} z_{n}\right| \leqq 3\left|\operatorname{Re} z^{\prime}\right|
$$

and if $\left|\operatorname{Re} z^{\prime}\right|<\left|\operatorname{Re} z_{n}\right|$ using (2. 24)

$$
\left|\operatorname{Im} z_{n}\right| \leqq\left|\operatorname{Im} z^{\prime}\right| .
$$
So we may for example conclude from Theorem 2.2. that the only function $u \in L_{\mathrm{loc}}^{1}\left(\mathbb{R}^{n}\right)$
with

$$
u(x)=0 \text { if } x_{n}<0 \quad \text { and } \int_{|y| \leqq 1} u(x+y) d y=0, \quad x \in \mathbb{R}^{n},
$$

is the function $u=0$.

Using the asymptotic properties of the Bessel function and the fact that the set of real zeros of $J_{\frac{n}{2}}$ is unbounded we may deduce that $\mu$ is invertible but not $C^{x}$-elliptic in the sense of Ehrenpreis [2]. Hence we have found an example of a convolutor $\mu$ showing that the non-existence of characteristic vectors does not imply $C^{x}$-ellipticity. For linear partial differential operators with constant coefficients, however, it is even true that (analytic-)ellipticity is equivalent to the non-existence of real characteristics vectors (see e.g. Hörmander [7]).

\section{Uniqueness in convex domains}

In this section we derive a refinement of Theorem 2. 2. for differential-difference equations with constant coefficients in convex domains. This will extend Theorem 5.3.3. in Hörmander [7].

Proposition 3. 1. Let $n>1$ and let $0 \neq \mu \in \mathscr{E}^{\prime}\left(\mathbb{R}^{n}\right)$. The set of non-characteristic vectors for $\mu$ is open in $\dot{R}^{n}$.

Proof. Let $N^{0} \in \mathbb{R}^{n}$ be a non-characteristic vector for $\mu$. Choose $\xi_{1}, \ldots, \xi_{n-1} \in \mathbb{R}^{n}$ such that the $\xi_{j}$ and $N^{0}$ span $\mathbb{R}^{n}$. If $N \in \dot{R}^{n}$ is close to $N^{0}$ then the $\xi_{j}$ together with $N$ still span $\mathbb{R}^{n}$. More precisely, there exist $a^{\prime} \in \mathbb{R}^{n-1}$ and $x \in \mathbb{R}, x \neq 0$, such that every $z \in \mathbb{C}^{n}$ which has coordinates $\left(z^{\prime}, z_{n}\right)$ with respect to the basis $\left(\xi_{1}, \ldots, \xi_{n-1}, N^{0}\right)$ has coordinates $\left(z^{\prime}+a^{\prime} z_{n}, \alpha z_{n}\right)$ with respect to the basis $\left(\xi_{1}, \ldots, \xi_{n-1}, N\right)$. A look at Definition 2. 1. shows that $N$ is a non-characteristic vector for $\mu$. Hence Proposition 3. 1. is proved. 
Let $0 \neq \mu \in \mathscr{E}^{\prime}\left(\mathbb{R}^{n}\right)$ and let $\Omega$ be a convex, open subset of $\mathbb{R}^{n}$. Then the convolution $\mu * u$ where $u \in \mathscr{L}^{\prime}(\Omega)$ is defined in

$$
\Omega_{\mu}=\{x ; x-y \in \Omega \text { when } y \in \operatorname{ch} \operatorname{supp} \mu\} .
$$
Here we denote by $\operatorname{ch} A$ the convex hull of a subset $A \subset \mathbb{R}^{n} . \Omega$ will be called $\mu$-round
if and only if

$$
\Omega=\Omega_{\mu}-\operatorname{ch} \operatorname{supp} \mu \text {. }
$$

Any open and convex subset of $\mathbb{R}^{n}$ is clearly $P(D) \delta$-round when $P$ is a non-zero polynomial. Let $\because$ be real and let $N \in \dot{R}^{n}$. The half-space $\{x ;\langle x, N\rangle \geqq \gamma\}$ will be called characteristic for $\mu$ if and only if its inward normal $N$ is a characteristic vector for $\mu$.

Theorem 3. 2. Let $0 \neq \mu \in \mathscr{\delta}^{\prime}\left(\mathbb{R}^{n}\right)$ with finite support. Let $\Omega^{1}$ and $\Omega^{2}$ be convex. open and $\mu$-round subsets of $\mathbb{R}^{n}$ with $\Omega^{1} \subset \Omega^{2}$. Assume that every characteristic half space interse'ting $\Omega^{2}$ also meets $\Omega^{1}$. Then ever. $u \in \mathscr{Z}^{\prime}\left(\Omega^{2}\right)$ satisfing the equation $\mu * u=0$ in $\Omega_{\mu}^{2}$
and tanishing in $\Omega^{1}$.

Prooff. (Compare the proof of Theorem 5.3.3. in Hörmander [7] and the proof of Theorem 2. 2. above.) Let $u \in C^{*}\left(\Omega^{2}\right)$ be given with

$$
u=0 \quad \text { in } \Omega^{1} \text { and } \mu * u=0 \text { in } \Omega_{\mu}^{2} .
$$

We shall show that $u=0$ in $\Omega_{\mu}^{2}$. In view of the standard regularization technique with Dirac
sequences this suffices to prove the theorem.

Let $r_{2}$ be a point in $\Omega_{\mu}^{2}$. Choosing a point $r_{1} \in \Omega_{\mu}^{1}$ (note that $\Omega_{\mu}^{1}$ is not empty since $\Omega^{1}$ is $\mu$-round) we denote by $K_{r}$ the set of all points at distance at most $\varepsilon$ from the line
segment between $l_{1}$ and subset of $\Omega_{a}^{2}$ and $\left\{x ;\left|, l_{2} l_{1}\right|<\delta\right.$ be a positive number so small that $K_{\delta}$ is a compact with ( ${ }^{1}$ boundary so that every characteristic half the set $(1)=(1)-$ ch $\operatorname{supp} \mu$. In fact icteristic half space intersecting $K_{\delta}$-ch supp $\mu$ also meets characteristic vector for $\mu$. we fact, if we let $x^{0} \in K_{\delta}-\operatorname{ch} \operatorname{supp} \mu$ and let $N^{0} \in \dot{R}^{n}$ be a such that $\left\langle x^{1}-x^{0} . x^{0}\right\rangle \geq 0$. Since $\Omega^{1}$. using the assumption of Theorem 3. 2., $x^{1} \in \Omega^{1}$ $\Sigma^{\prime} \subset \Omega_{\mu}^{1}$ such that - -ch supp $\mu$ me $\Omega^{1}$ is $\mu$-round we therefore obtain an open ball meets every half space with inward normalf space $\left\{x ;\left\langle x-x^{0}, N^{0}\right\rangle \geqq 0\right\}$ and consequently Proposition 3. 1. and the Borel-Lebesgue close to $N^{0}$ containing a point near $x^{0}$. By can therefore be constructed by taking lommat a set ()$^{\prime} \subseteq \Omega_{\mu}^{1}$ with the required properties balls $\Sigma \Subset \Omega_{u}^{1}$.

Now let $\omega_{t}^{\prime}, 0 \leqq t \leqq 1$, be the convex hull of $\omega^{\prime}$ and the open ball $\Sigma_{t}$ with radius $\delta$ We center at $(1-t) l_{1}+t y_{2}$. It is geometrically evident that the boundary of $\omega_{t}^{\prime}$ is $C^{1}$. $v_{0} \subset \Omega^{1}$. Let $\tau$ be the supremum of $\omega_{t}=\omega_{t}^{\prime}-\operatorname{ch} \operatorname{supp} \mu$ when $0 \leqq t \leqq 1$. This is true when $t=0$ because $u=0$ in (a. We shall show that $u=0$ in a neighbour 0 and 1 such that $u=0$ in $\omega_{t}$. Then course prove our claim. 
Let $x^{0}$ be a boundary point of $\omega_{\tau}$. If $x^{0} \in \Omega^{1}$, then clearly $u=0$ in a neighbourhood of $x^{0}$. Now assume that $x^{0} \notin \Omega^{1}$. Then we can write $x^{0}=x^{1}-y$ with $y \in \operatorname{ch} \operatorname{supp} \mu$ and $x^{1} \in \hat{c} \omega_{\tau}^{\prime}$ such that the outward normal $N$ of $\hat{c} \omega_{\tau}^{\prime}$ in $x^{1}$ is a non-characteristic vector for $\mu$. In fact, if $\pi$ is a characteristic half space not intersecting $\omega_{\tau}$, then $\pi \cap \omega=0$ which implies that $\pi \cap\left(K_{\delta}-\right.$ ch $\left.\operatorname{supp} \mu\right)=\emptyset$. Hence $\pi \cap\left(\bar{\Sigma}_{\tau}-\operatorname{ch} \operatorname{supp} \mu\right)=0$, so that

$$
\pi \cap \bar{\omega}_{\tau}=\pi \cap \bar{\omega} \subset \Omega^{1}
$$

Assuming without loss of generality that $x^{0}=0$ and $N=(0, \ldots, 0,1)$ and recalling that the boundary of $\omega_{\tau}^{\prime}$ is $C^{\mathbf{l}}$ we have for any $\varepsilon>0$

$$
\left\{x ;|x|<\delta(\varepsilon), x_{n}<-\varepsilon\left|x^{\prime}\right|\right\} \subset()_{\tau} .
$$

Since $N$ is non-characteristic we have for some $b>0$ and every $\delta>0$

$$
\mu(-z-\zeta) \neq 0 \quad \text { if } \quad z \in E(b, \delta),|z| \geqq C(\delta),|\zeta|<1 .
$$

By (3.1) we may choose $a>0$ such that

$$
\begin{gathered}
K=\left\{x ;\left|x^{\prime}\right| \leqq a, \quad x_{n}=-a(4 b)^{-1}\right\} \Subset \omega_{\tau} . \\
\left\{x ;\left|x^{\prime}\right| \leqq a,-a(4 b)^{-1} \leqq x_{n} \leqq\left(a^{2} / 2-\left|x^{\prime}\right|^{2}\right)(2 a b)^{-1}\right\} \Subset Q^{2} .
\end{gathered}
$$

Let $H$ be the supporting function of $K$, i.e. $H(\eta)=a\left|\eta^{\prime}\right|-a(4 b)^{-1} \eta_{n}, \eta \in \mathbb{R}^{n}$. Let $a>0$ and let $v \in \mathscr{E}^{\prime}\left(\Omega^{2}\right)$ be given such that

$$
|\hat{v}(z)| \leqq C \exp (H(\operatorname{Im} z)+\varepsilon|=|) \quad \text { if } \quad z \in C^{n} \backslash E(b, b \varepsilon(a) .
$$

We are now going to prove that $v(u)=0$. This will be done along the lines of the proof of Theorem 2. 2. That $\mu$ has finite support means that its Fourier transform $\mu$ is an exponential-polynomial. But for these it is known (see e.g. Grudzinski [5]. Theorem 10) that for some constant $C>0$

$$
|\hat{\mu}(-z)| \geqq C \exp H_{\breve{\mu}}(\operatorname{Im} z)
$$

if $z \in \mathbb{C}^{n}$ has distance at least 1 to the set of zeros of $\tilde{\hat{\mu}}$. Hence it follows from (3.2) when taking $\delta=(2 a)^{-1} b$; that $(3.4)$ is valid for all sufficiently large $z \in E\left(b \cdot(2 a)^{-1} b \varepsilon\right)$. We may therefore choose a $C^{x}$-function $\varphi$ which is equal to one in a neighbourhood of the set of zeros of $\tilde{\hat{\mu}}$ and such that $|\overline{\hat{\alpha}} \varphi|$ is bounded in $\bar{C}^{n}$ and the estimates (3.3) and (3.4) are satisfied in supp $\varphi$ and supp $(1-\varphi)$. respectively. Defining the $(0.1)$-form $g$ according to (2. 14) we then have

$$
|g(z)| \leqq C \exp \left(H(\operatorname{Im} z)-H_{\breve{\mu}}(\operatorname{Im} z)+\varepsilon|=|\right) . z \in \mathbb{C}^{n} .
$$

Let $H^{\prime}$ denote the supporting function of a convex set $K^{\prime} \Subset(1)^{\prime}$ with $K \subset K^{\prime}-$ ch supp $\mu$. Then $H \leqq H^{\prime}+H_{\breve{\mu}}$ and hence

$$
|g(z)| \leqq C \exp \left(H^{\prime}(\operatorname{Im} z)+s|=|\right),=\in \mathbb{C}^{n} .
$$

Since $H^{\prime}(\mathrm{Im} \cdot)$ is plurisubharmonic (whereas $\left(H-H_{\breve{\mu}}\right)(\mathrm{Im} \cdot)$ need not be!) we obtain. using Theorem 4. 4. 2. in Hörmander [8], a solution to the equation $\overline{\hat{c}} v=g$ with

$$
\int_{c_{n}}|v(z)|^{2} \exp \left(-2 H^{\prime}(\operatorname{Im} z)-3 \varepsilon|z|\right) d \lambda(z)<+x \text {. }
$$


The functions $S$ and $T$ defined by (2.10) and (2.11) are entire analytic, and combining (3.3), (3.4). (3.5) and the Paley-Wiener estimates for $\hat{v}$ and $\hat{\mu}$ we obtain

$$
S \exp \left(-H^{\prime}(\operatorname{Im} \cdot)-H_{\breve{\mu}}(\operatorname{Im} \cdot)-2 \varepsilon|\cdot|\right) \in L^{2}\left(\mathbb{C}^{n}\right)
$$

and

$$
T \exp \left(-\max \left(H_{v}(\operatorname{Im} \cdot)-H_{\breve{\mu}}(\operatorname{Im} \cdot), H^{\prime}(\operatorname{Im} \cdot)\right)-2 \varepsilon|\cdot|\right) \in L^{2}\left(\mathbb{C}^{n}\right) .
$$

With the help of Cauchy's integral formula we can pass from the $L^{2}$ - to the sup-estimates

$$
\begin{array}{ll}
|S(z)| \leqq C \exp \left(H_{1}(\operatorname{Im} z)+2 \varepsilon(|\operatorname{Re} z|-2|\operatorname{Im} z|)\right), & z \in \mathbb{C}^{n}, \\
|T(z)| \leqq C \exp \left(H_{2}(\operatorname{Im} z)+2 \varepsilon(|\operatorname{Re} z|-2|\operatorname{Im} z|)\right), & z \in \mathbb{C}^{n} .
\end{array}
$$

Here we have introduced the supporting functions

$$
\begin{aligned}
& H_{1}(\eta)=H^{\prime}(\eta)+H_{\breve{\mu}}(\eta)+6 \varepsilon|\eta|, \quad \eta \in \mathbb{R}^{n}, \\
& H_{2}(\eta)=\max \left(H_{v}(\eta)-H_{\breve{\mu}}(\eta), H^{\prime}(\eta)\right)+6 \varepsilon|\eta|, \quad \eta \in \mathbb{R}^{n} .
\end{aligned}
$$

Choose $\varepsilon>0$ such that

$$
\left\{x ;\langle x, \eta\rangle \leqq H_{1}(\eta), \eta \in \mathbb{R}^{n}\right\} \Subset \omega_{\tau} \text { and }\left\{x ;\langle x, \eta\rangle \leqq H_{2}(\eta), \eta \in \mathbb{R}^{n}\right\} \Subset \Omega_{\mu}^{2} .
$$

Applying Theorem 2.5. as in the proof of Theorem 2.2. with $F_{1}:=S, F_{2}:=T$ and $G_{2}:=\mu$ we obtain distributions $\sigma_{t}, \tau_{t} \in \mathscr{E}^{\prime}\left(\mathbb{R}^{n}\right), 0<t<1$, such that $\sigma_{t}+\check{\mu} * \tau_{t} \rightarrow v$ in $\mathscr{E}^{\prime}\left(\Omega_{\mu}^{2}\right)$ as $t \rightarrow 0$ and $\operatorname{supp} \sigma_{t} \Subset \omega_{\tau}$, supp $\tau_{t} \Subset \Omega_{\mu}^{2}$. Hence $v(u)=0$. Recalling that $v$ was an arbitrary in a neighbourhood of $x^{0}$. So (3.3) it now follows from Lemma 2.3. that $u$ vanishes hence $u=0$ in $\omega_{1}$. Since $v_{2} \in \Omega^{2}$ wase proved that $u=0$ in a neighbourhood of $\bar{\omega}_{\tau}$ and of Theorem 3. 2. is complete. $\quad \mu$ was arbitrary and since $\Omega^{2}=\Omega_{\mu}^{2}-$ ch supp $\mu$ the proof

Remark. It is easily seen by inspecting the foregoing proof that Theorem 3.2. is true not only for distributions with finite support but also for distributions $\mu \in \mathscr{E}^{\prime}\left(\mathbb{R}^{n}\right)$
which satisfy for every $\varepsilon>0$

$$
|\hat{\mu}(z)| \geqq C(\varepsilon) \exp \left(H_{\mu}(\operatorname{Im} z)-\varepsilon|z|\right) \quad \text { if } \quad d(z) \geqq 1+\varepsilon|z|, z \in \mathbb{C}^{n} .
$$

However, if $\mu$ is the characteristic function of the unit ball in $\mathbb{R}^{n}, n>1$ (recall section 2, Example) then using the estimate $|\hat{\mu}(z)| \leqq c \exp \left(\left|\operatorname{Im}\langle z, z\rangle^{\frac{1}{2}}\right|\right)$ and the reality of the zeros of $\left|I \mathrm{~m} z_{0}\right|>\left|\operatorname{Re} z_{0}\right|=1$. $J_{\frac{n}{2}}$ one can see by considering the line $\left\{t z_{0} ; t \in \mathbb{R}\right\}$ for $z_{0} \in \mathbb{C}^{n}$ with pointed out to me by $O$. v. Grudzinski.
. (3.6) does not hold for $\mu$. This example was

\section{Non-uniqueness of the Cauchy problem}

For a non-zero polynomial $P$ let $m$ denote its degree and $P_{m}$ its principal part.

Proposition 4. 1. Let $N \in \dot{R}^{n}$ and let $P$ be a non-zero polynomial and $v \in \mathscr{E}^{\prime}\left(\mathbb{R}^{n}\right)$ with supp $v \in\{x:\langle x, N\rangle>0\}$. Define $\mu=P(D) \delta+v$. Then $N$ is a characteristic vector for $\mu$ if
and only if $P_{m}(N)=0$. 
Proof. Let $\xi \in \mathbb{R}^{n}$. First note that since $H_{v}(-N)<0$ we have by the Paley-Wiener theorem with some constants $A>0, \delta>0$

$$
|\hat{v}(z+t \xi)| \leqq \exp \left(\delta \operatorname{Im} z_{n}+A\left|\operatorname{Im} z^{\prime}\right|+A|t|+A \log (2+|z|)\right)
$$

for $z \in \mathbb{C}^{n}$ and $t \in \mathbb{C}$ satisfying $\operatorname{Im} z_{n} \leqq 0,|t|=O(|z|)$.

Here coordinates are chosen such that $N=(0, \ldots, 0,1)$. Since the degree of the polynomial $P_{m}-P$ is at most $m-1$ we therefore obtain for $\hat{\mu}=P+\hat{v}$ with some positive constant $C$

$$
\left|P_{m}(z+t \xi)-\hat{\mu}(z+t \xi)\right| \leqq C(1+|z|)^{m-1}
$$

if $z \in \mathbb{C}^{n}$ and $t \in \mathbb{C}$ satisfy

$$
|t| \leqq|z| \text { and }-\operatorname{Im} z_{n} \geqq C\left(\left|\operatorname{Im} z^{\prime}\right|+|t|+\log (2+|z|)\right) .
$$

If $P_{m}(N) \neq 0$ we have with some positive constants $C_{1}$ and $C_{2}$

$$
\left|P_{m}(z)\right| \geqq C_{1}|z|^{m} \quad \text { if } \quad\left|z_{n}\right| \geqq C_{2}\left|z^{\prime}\right| .
$$

In fact, since $z$ belongs to a given conic neighbourhood of $N$ if $\left|z^{\prime}\right| /\left|z_{n}\right|$ is sufficiently small and since $P_{m}\left(0, \ldots, 0, z_{n}\right) \neq 0$ if $z_{n} \neq 0$, (4.2) follows from the continuity and homogeneity of $P_{m}$. Combining (4.1) for $t=0$ and (4. 2) we have $\left|P_{m}(z)-\hat{\mu}(z)\right|<\left|P_{m}(z)\right|$ and hence $\hat{\mu}(z) \neq 0$ if $\left|z_{n}\right| \geqq C_{2}\left|z^{\prime}\right|,-\operatorname{Im} z_{n} \geqq C\left(\left|\operatorname{Im} z^{\prime}\right|+\log (2+|z|)\right)$ and $|z|$ large. Hence $N$ is a non-characteristic vector for $\mu$.

Now assume that $P_{m}(N)=0$. Choose $\xi$ such that $P_{m}(\xi) \neq 0$. Denote by $d, 1 \leqq d \leqq m$, the order of the zero $w=0$ of the non-zero polynomial $P_{m}(N+w \xi)$ in the complex variable $w$. Hence we have with some positive number $\delta$

$$
\left|P_{m}(s N+t \xi)\right|>\delta|s|^{m-d}|t|^{d} \quad \text { if } \quad|t|<\delta|s|, \quad t, s \in \mathbb{C} .
$$

Replacing z by $s N$ in (4.1) we therefore obtain with some constant $C>0$

$$
\left|P_{m}(s N+t \xi)-\hat{\mu}(s N+t \xi)\right|<\left|P_{m}(s N+t \xi)\right|
$$

if $s, \mathrm{t} \in \mathbb{C}$ satisfy $|s|>C,|t|<C|s|^{1-1 / d}$ and $-\operatorname{Im} s \geqq C(|t|+\log (2+|s|))$. In view of Rouchés theorem we may now conclude from (4.3) that we can find for every sufficiently large $\sigma>0$ a complex number $t$ such that $\hat{\mu}(-i \sigma N+t \xi)=0$ and $|t|\left\langle C^{\prime}|\sigma|^{1-1 d}\right.$. It is easily checked that $N$ is a characteristic vector for $\mu$ in the sense of Definition 2. 1. This completes the proof of the proposition.

The following non-uniqueness theorem generalizes Theorem 5. 5. 2. in Hörmander [7]. The new feature in our theorem is that we do not rely on the Puiseux expansion but instead use the holomorphy of certain functions symmetric in some of the zeros of $\hat{\mu}$. Possibly this idea is flexible enough to prove non-uniqueness for more general convolution equations than those considered in Theorem 4. 2.

Theorem 4. 2. Let $N \in \dot{R}^{n}$ and let $P$ be a non-zero polynomial and let $v \in \mathscr{E}^{\prime}\left(\mathbb{R}^{n}\right)$ with $\operatorname{supp} v \Subset\{x,\langle x, N\rangle>0\}$. Assume that $N$ is a characteristic vector for $\mu=P(D) \delta+v$. Then there exists a function $u \in C^{\infty}\left(\mathbb{R}^{n}\right)$ with $0 \in \operatorname{supp} u$, supp $u \subset\{x ;\langle x, N\rangle \geqq 0\}$ and $\mu * u=0$ in $\mathbb{R}^{n}$. 
Proof. We have $P_{m}(N)=0$ by Proposition 4. 1. Let $\xi \in \dot{R}^{n}, P_{m}(\xi) \neq 0$, and $d \geqq 1$ be as in the proof of Proposition 4.1. Recall that (4.3) is valid then. From (4.3) and Rouchés theorem it now follows that there are positive constants $\rho$ and $C$ such that for every $s \in \mathbb{C}$ with

$$
-\operatorname{Im} s>C\left(1+|s|^{1-1 / d}\right)
$$

the holomorphic function $g$ defined by $g(t)=\hat{\mu}(s N+t \xi), t \in \mathbb{C}$, has exactly $d$ zeros $t_{j}(s), j=1, \ldots, d$ (counted with multiplicity), satisfying

$$
\left|t_{j}(s)\right|<\rho|s|^{1-1 / d} .
$$

Hence it follows from the argument principle that for every $x \in \mathbb{R}^{n}$ and every $s$ with (4.4)

$$
\sum_{j=1}^{d} \exp \left(i\left\langle x, t_{j}(s) \xi\right\rangle\right)=(2 \pi i)^{-1} \int e^{i t\langle x, \xi\rangle}\left(g^{\prime}(t) / g(t)\right) d t
$$

when integrating over the contour $|t|=\rho|s|^{1-1 / d}$, and therefore the left-hand side in (4.6) is a holomorphic function in $s$ in the domain of all $s$ satisfying (4. 4).

Now choose $1>\gamma>1-1 / d$ and define for large positive $\sigma$

$$
u(x)=\int_{\Gamma_{\sigma}} \sum_{j=1}^{d} \exp \left(i\left\langle x, s N+t_{j}(s) \xi\right\rangle-(i s)^{\gamma}\right) d s, x \in \mathbb{R}^{n},
$$

where $\Gamma_{\sigma}$ is the contour defined by

$$
-\operatorname{Im} s=\sigma\left(1+|\operatorname{Re} s|^{2}\right)^{(1-1 / d) / 2}
$$

and $(i s)^{*}$ is defined so that it is real and positive when $s$ is negative imaginary. The in a fixed bounded set we have in view of $(4.5)$ for large $s$
in

$$
\begin{aligned}
\operatorname{Re}\left(i\left\langle x, s N+t_{j}(s) \xi\right\rangle-(i s)^{\gamma}\right) & \leqq-\operatorname{Im} s\langle x, N\rangle+\rho|x||\xi||s|^{1-1 / d}-|s|^{\gamma} \cos (\pi \gamma / 2) \\
& \leqq-\operatorname{Im} s\langle x, N\rangle-2 C|s|^{\gamma}
\end{aligned}
$$

Hence for $s$ satisfying (4. 8) with $\sigma$ large we have

$$
\operatorname{Re}\left(i\left\langle x, s N+t_{j}(s) \xi\right\rangle-(i s)^{\gamma}\right) \leqq-C|s|^{\gamma} .
$$

This estimate also shows that the integral is uniformly convergent after arbitrary diffe(4. 8) and (4. 9) that $u(x)=0$ if $\langle x, N\rangle<0$.

Now $u(x)=d u(\langle x, N\rangle)$ when $\langle x, \breve{\zeta}\rangle=0$, where we use the notation

Reasoning in the same way as in the proof of Theorem 5.2.2. in Hörmander [7] to
which we refer the reader for further details we obtain $0 \in \operatorname{supp} v$ and hence $0 \in$ supp $u$
This proves Theorem 4.2. $v(t)=\int_{\Gamma_{\sigma}} \exp \left(i s t-(i s)^{\gamma}\right) d s, t \in \mathbb{R}$. 


\section{References}

[1] S. Bochner, Lectures on Fourier integrals, Ann. of Math. Studies 42 (1959).

[2] L. Ehrenpreis, Solutions of some problems of division. IV, Amer. J. Math. 82(1960)). 522.588.

[3] L. Ehrenpreis, Solutions of some problems of division. V, Amer. J. Math. 84 (1962). $324 \quad 348$.

[4] L. Ehrenpreis, Fourier Analysis in Several Complex Variables, New York 1970.

[5] O. v. Grud-inski, Einige elementare Ungleichungen für Exponentialpolynome, Math. Ann. 221 (1976). 9_ -34

[6] S. Hansen, Zum Cauchyproblem für Convolutoren, K iel 1977.

[7] L. Hörmander, Linear Partial Differential Operators, Berlin-Heidelberg-New York 1969.

[8] L. Hörmander, An Introduction to Complex Analysis in Several Variables. Amsterdam-London 1973.

[9] L. Hormander, Convolution Equations in Convex Domains, Invent. Math. 4(1968), 306-317.

[10] G. N. Watson, A Treatise on the Theory of Bessel Functions, Cambridge 1966.

Fachbereich 17 der Gesamthochschule. Warburger Straße 100, D-4790 Paderborn

Eingegangen 12. Februar 1979 